\title{
Prognostic value of telomere attrition in patients with aplastic anemia
}

\author{
Phillip Scheinberg
}

Received: 7 April 2013/Accepted: 10 April 2013/Published online: 1 May 2013

(C) The Japanese Society of Hematology 2013

\begin{abstract}
The decision to pursue hematopoietic stem cell transplantation or immunosuppression as first therapy in severe aplastic anemia is currently based on age and availability of a histocompatible donor. The ability to predict hematologic response, relapse and clonal evolution could improve treatment allocation. In the past 15 years, telomeres have been implicated in clinical diseases such as aplastic anemia, pulmonary fibrosis, cirrhosis and cancer development. The clinical relevance of varying telomere lengths (TL) and/or mutations in genes of the telomerase complex (TERC, TERT) is evolving in aplastic anemia. A large retrospective analysis suggests that baseline TL associate with late events of hematologic relapse and clonal evolution in aplastic anemia patients treated initially with anti-thymocyte globulin-based therapy. Further laboratory experiments propose possible mechanistic insight into genomic instability of bone marrow cells derived from patients with critically short telomeres and/or mutation in telomerase genes. The possibility of modulating telomere attrition rate with sex hormones could positively affect clonal evolution rates in humans. This review will summarize studies in marrow failure that explore the association between telomeres and aplastic anemia outcomes.
\end{abstract}

Keywords Aplastic anemia - Telomeres - Relapse · Clonal evolution $\cdot$ Pancytopenia $\cdot$ Immunosuppressive therapy

\section{P. Scheinberg $(\square)$}

Hematology Service, Oncology Center, Hospital São Jose,

Beneficência Portuguesa, Rua Martiniano de Carvalho,

951, São Paulo, SP 01321-001, Brazil

e-mail: phillip.scheinberg@ hospitalsjose.org.br

\section{Introduction}

Up to 40 years ago, severe aplastic anemia (SAA) was associated with a high fatality rate 1-2 years after the diagnosis. However, the majority of patients nowadays are expected to be alive long-term following the diagnosis of SAA. The treatment modalities that contributed to this success are hematopoietic stem cell transplantation (HSCT) and immunosuppressive therapy (IST). Although the toxicities vary between these two approaches, longterm outcome with either therapy is comparable [1]. In general, HSCT from a matched related sibling donor is applied to children and adults up to the age of 40 as first therapy, while IST is often employed in older patients (over 40) or when a sibling donor is not available [1,2]. Thus, the decision to pursue either therapy relies nowadays on age and matched related donor availability. Comorbidities can play a role in the decision-making, however in general younger patients with SAA usually do not have other significant underlying illnesses that preclude a matched related HSCT.

HSCT is curative in the majority of patients, and IST is often associated with hematologic response rates that have ranged from 60 to $75 \%$ across many large studies in the United States, Europe, and Japan [3]. However, graft rejection, infections, and more importantly, graft-versushost disease (GVHD) limit the success of HSCT, while unresponsiveness, relapse and clonal evolution to myelodysplasia (MDS) limit the success of IST. Unfortunately, there are no practical robust marker(s) that can predict these important outcomes following HSCT or IST which could better risk-stratify patients allowing for a more rational treatment allocation. There have been many reports towards this goal; however, none has been widely adopted yet into clinical practice. In this review, some of 
these efforts will be summarized which could allow for better decision-making algorithms in the future.

\section{Important outcomes following IST}

The important outcomes to be considered following IST are hematologic response, relapse and clonal evolution to MDS and survival. In general, response can be anticipated in $2 / 3$ of cases, relapse in about $1 / 3$ of responders and evolution in 10-15\% long-term $[4,5]$. The standard IST regimen in SAA is with the combination of horse antithymocyte globulin (h-ATG) and cyclosporine (CsA). This regimen has been shown superior as first therapy to alternative regimens such as rabbit ATG or alemtuzumab in randomized studies $[6,7]$. The most important predictor for long-term survival is hematologic response at 3-6 months following h-ATG/CsA [4]. Relapses, in general, are not usually associated with a dire outcome since most do respond to more immunosuppression with CsA or another course of IST [4, 8, 9]. Clonal evolution, however, remains a significant problem especially with the appearance of a monosomy 7 karyotype or evidence of high-grade dysplastic findings with increase in blasts $[1,10]$.

\section{Can hematologic response be predicted?}

There have been several attempts to predict hematologic response in SAA following IST. Some research laboratory findings that reflect the pathophysiology of SAA, such as the increased ratio of activated $T$ cells, increased interferon- $\gamma$ expression in bone marrow and peripheral $\mathrm{T}$ cells, increased expression of heat shock protein, and the presence of small numbers of aneuploid bone marrow cells have been proposed as useful in prognosis; however, these methods, which are not currently either generally available or applied, require validation [11-14]. A more simplified approach has been to associate the presence of a paroxysmal nocturnal hemoglobinuria $(\mathrm{PNH})$ clone with response. The presence of a PNH clone at baseline has been suggested as a marker of a favorable response to IST in adults by using a more sensitive flow cytometric assays that improved detection of small clones compared to the more traditional Ham's test [15]. A correlation of a PNH clone with response, however, has not been confirmed in other studies [16, 17].

Quantitative assessment of reticulocyte count has been proposed as marker for response, maybe serving as an indirect measurement of marrow reserve $[16,18,19]$. In a large retrospective study, those with a higher baseline reticulocyte count had a higher probability of response and survival compared to those with a lower reticulocyte at baseline. This parameter has been confirmed in some reports but not in others [20, 21]. Of interest is that robust reticulocyte recovery at 3 months following IST has been associated with better survival outcomes and baseline reticulocyte count associated with response in a study of a thrombomimetic, eltrombopag, in refractory SAA $[4,22]$. The absolute neutrophil count appears to be associated with short-term mortality in many reports, but this observation was not confirmed in a German experience in children [23, 24].

Age has been a more consistent defining predicting parameter across many studies. In general, children do have a higher response rate compared to older patients following IST [16, 25-28]. The response rate in children is usually reported in the 70-80\% range with excellent survival among responders, compared to a 60-70 \% response rate in younger adults and a 50-60\% rate in those over the age of 40-50 years of age. Although there has not been a randomized study comparing the more intense IST with h-ATG/CsA to HSCT, the latter is the preferred modality in children and young adults with a histocompatible donor, while the former is an excellent alternative among those without a related donor.

\section{Can hematologic relapse and clonal evolution be predicted?}

These two late events can be associated with morbidity and mortality in patients in follow-up. The mechanism involved in these complications are poorly understood however continuing subclinical immune destruction of stem cells or hematopoietic exhaustion has been proposed for relapse, and selection of pre-malignant clones resistant to apoptosis, proliferative advantage, and genomic instability proposed for evolution. In practice, it is difficult to assess these parameters quantitatively or qualitatively.

Defect in telomere repair mechanisms has been implicated in inherited forms of marrow failure such as dyskeratosis congenita, which are generally diagnosed at a young age after a presentation of aplastic anemia associated with physical abnormalities early in life [29]. Telomeres represent the ends of chromosomes which progressively shorten with each cellular division resulting in its shortening. A critical short telomere length (TL) results in a cessation of replication and cell death. To counter this erosion, the telomerase complex adds short nucleotide repeats (TTAGGG) to the ends of chromosomes (telomeres) which each division slowing the attrition rate. This complex is composed of an RNA template that is encoded by TERC and a catalytic subunit encoded by $T E R T$. In general, expression of telomerase is low in most somatic cells however, in hematopoietic stem cells and lymphocytes which have a high proliferative capacity 
expression is high. Mutations in TERC and TERT have been identified in $5-10 \%$ of acquired SAA cases who present later in life without physical abnormalities [30].

Early reports suggested that telomeres were short in up to one-third of patients with SAA [31, 32]. Initially, this occurrence was presumed to be secondary to hematopoietic "stress". However, the discovery of loss-of-function mutations in genes of the telomerase complex (TERC, $T E R T$ ) established a genetic etiology for telomere attrition in some patients with marrow failure who did not have the stigmata associated with an inherited bone marrow failure syndrome [33, 34]. In the past 15 years, the relationship between TL, genetic defects in the telomerase complex and marrow failure has been an area of great interest. In a large retrospective analysis at the National Institutes of Health (NIH), 183 patients with SAA treated on sequential prospective ATG-based immunosuppression protocols had their outcomes correlated with age-adjusted TL of peripheral blood leukocytes at baseline measured prior to study entry [35]. The main outcomes included in the analysis were hematologic response at 6 months, relapse, clonal evolution, and survival. For this analysis, the TL was divided into 4 quartiles with the shortest lengths represented in the first quartile, while the longer lengths represented in the fourth quartile. There was no relationship observed in this study between baseline TL and hematologic response at 6 months. The response rate for patients with TL in the first quartile was $57 \%$ [95\% confidence interval (CI) 42-71], second quartile $54 \%$ (95\% CI 39-69), third $60 \%$ (95\% CI 45-75), and fourth quartile $57 \%$ (95\% CI 42-71).

However, a relationship between TL with relapse and clonal evolution was observed [35]. When measured as a continuous variable, TL inversely correlated with the probability of relapse [hazard ratio (HR) 0.16 (95\% CI $0.03-0.69) ; p=0.01]$. The risk for relapse progressively decreased from quartiles 1 (shortest) to 4 (longest). For clonal evolution, a higher rate of cytogenetic abnormalities was observed in those in quartile $1(25 \%, 95 \%$ CI 9-38) compared to quartiles 2-4 (8\%, $95 \%$ CI 3-13; $p=0.009$ ). More importantly, higher risk cytogenetic defects such as monosomy 7 and complex cytogenetics were noted in quartile $1(19 \%, 95 \%$ CI 4-32) compared to those in quartiles $2-4(5 \%, 95 \% \mathrm{CI}, 0.5-8, p=0.002)$. Overall survival also differed at 6 years between those with TL in quartile $1(66 \%, 95 \%$ CI 53-83) and quartiles 2-4 (84\%, $95 \%$ CI 77-91; $p=0.008)$. It is important to note that a genetic defect in TERC or TERT was identified in only 1 patient in this cohort. Thus, this study described a relationship between TL within the normal range and late events in SAA following ATG-based immunosuppression.

It is unlikely that $\mathrm{TL}$ is a simple biomarker for late events in SAA, but that it is also involved in the destabilization of the genome and participates in the etiopathogenesis of clonal evolution. A short TL has been widely associated with the development of human cancer in population-based studies and in pre-malignant inflammatory conditions such as Barrett's esophagus and inflammatory bowel disease [30, 36-40]. In a follow-up study by Calado et al. [41], bone marrow cells from patients in the NIH cohort were studied for the presence of monosomy 7 by fluorescent in vitro hybridization (FISH) at diagnosis. A strong inverse correlation was observed between average leukocyte TL and the percentage of monosomy 7 cells by FISH in the marrow $(r=-0.503$, $p=0.001)$. An increased frequency of telomere signal free ends (a marker for very short TL and genomic instability) was also noted in cultured bone marrow cells of patients with a lower average TL (lower quartiles) compared to those in the upper quartiles or healthy subjects. In addition, a significant proportion of numeric aberrations and structural chromosomal abnormalities were observed in cultured bone marrow cells from patients with the shorter TL compared with patients with longer telomeres or to healthy controls [41]. Finally, cultured telomerase-mutant marrow cells from individuals identified in family studies of patients with aplastic anemia and telomerase mutations developed an increase proportion of aneuploid cells when compared to bone marrow cells of healthy individuals under same culture conditions. These observations support the notion that short and dysfunctional telomeres restrict the proliferation of normal hematopoietic stem cells and lead to the selection of genomic unstable cells.

Although the average TL is often used in correlative studies, it is the shortest telomeres that contribute to chromosomal instability rather than the average TL [42]. A more sensitive assay of single telomere length assay (STELA) using a single PCR reaction to amplify chromosome-specific telomeres allows for the identification of critically short TL regardless of the average TL by qPCR [43]. In a small study presented in abstract form recently, very short TL by STELA was present in a higher proportion of SAA patients who progressed to MDS when compared with complete responders to immunosuppression [44]. Ongoing studies to better define the heterogeneity of TL by STELA and their clinical consequence could identify very short telomeres in a subpopulation of cells that pose a greater risk for clonal evolution in some aplastic anemia patients.

\section{Can telomere attrition rate be modulated in vivo?}

The historically low rate of clonal evolution in AA patients who recovered with androgens suggests therapeutic opportunity with these agents to decrease evolution rates long-term [45]. The possible role of a critical short TL and clonal evolution in AA raises the possibility that 
modulation of telomere attrition may reduce clonal events in humans. Exposure in vitro of human peripheral blood leukocytes and bone marrow cells to androgens has been shown to increase telomerase activity along with increased expression of TERT mRNA [46]. This observation was expanded in vivo where mice under certain hematopoietic stress had a reduced attrition rate when treated with testosterone compared to mice who received a corn oil control [47]. A recent case report in an aplastic anemia patient suggests that continued androgen use might be associated with hematologic improvements along with TL elongation of peripheral blood leukocytes [48]. Thus, the effect of androgen therapy on increasing telomerase activity might contribute to slowing telomere attrition, assisting in cell regeneration and ultimately prevent genomic destabilization. In order to address this modulatory possibility in humans, an ongoing clinical trial (clinicaltrials.gov NCT01441037) at the NIH is currently investigating the activity of danazol in patients with a telomere disorder associated with hematologic or pulmonary disease. The objective of this study is to determine if androgen therapy can reduce the telomere attrition rate in this patient population with a 2-year course of danazol.

In conclusion, biomarkers that correlate with hematologic response, relapse and clonal evolution would greatly enhance risk-stratification and allow for better treatment allocation in SAA. For example, younger patients with a low probability of response and high probability of late events might benefit more from an unrelated donor HSCT as first therapy, while older patients with a similar risk profile benefit more from a matched sibling donor HSCT. The association of TL and late events (relapse, clonal evolution) requires validation before entering decisionmaking algorithms in SAA. The possible role of short telomeres contributing to genomic instability and evolution to MDS opens the possibility of investigating agents that could modulate the attrition rate and hopefully reduce the rate of this complication. The clinical relevance of genetic defects in telomerase genes in patients who otherwise present with 'acquired' SAA is still evolving. Some patients with known mutations in TERT or TERC can respond to IST similarly as in non-mutants [49]. If a genetic defect is sufficient to define the pathogenesis in some cases of apparent acquired AA or it is a contributor to other pathophysiologic mechanisms of marrow destruction such as an aberrant immune system remains to be defined.

\section{References}

1. Scheinberg P, Young NS. How I treat acquired aplastic anemia. Blood. 2012;120:1185-96.
2. Scheinberg P. Aplastic anemia: therapeutic updates in immunosuppression and transplantation. Hematol Am Soc Hematol Educ Progr. 2012;2012:292-300.

3. Young NS, Calado RT, Scheinberg P. Current concepts in the pathophysiology and treatment of aplastic anemia. Blood. 2006;108:2509-19.

4. Rosenfeld S, Follmann D, Nunez O, Young NS. Antithymocyte globulin and cyclosporine for severe aplastic anemia: association between hematologic response and long-term outcome. JAMA. 2003;289:1130-5.

5. Bacigalupo A, Bruno B, Saracco P, et al. Antilymphocyte globulin, cyclosporine, prednisolone, and granulocyte colony-stimulating factor for severe aplastic anemia: an update of the GITMO/ EBMT study on 100 patients. European Group for Blood and Marrow Transplantation (EBMT) Working Party on Severe Aplastic Anemia and the Gruppo Italiano Trapianti di Midolio Osseo (GITMO). Blood. 2000;95:1931-4.

6. Scheinberg $\mathrm{P}$, Nunez $\mathrm{O}$, Weinstein $\mathrm{B}$, et al. Horse versus rabbit antithymocyte globulin in acquired aplastic anemia. N Engl J Med. 2011;365:430-8.

7. Scheinberg $\mathrm{P}$, Nunez $\mathrm{O}$, Weinstein $\mathrm{B}$, Scheinberg $\mathrm{P}, \mathrm{Wu} \mathrm{CO}$, Young NS. Activity of alemtuzumab monotherapy in treatmentnaive, relapsed, and refractory severe acquired aplastic anemia. Blood. 2012;119:345-54.

8. Tichelli A, Passweg J, Nissen C, et al. Repeated treatment with horse antilymphocyte globulin for severe aplastic anaemia. Br J Haematol. 1998;100:393-400.

9. Scheinberg P, Nunez O, Young NS. Retreatment with rabbit antithymocyte globulin and ciclosporin for patients with relapsed or refractory severe aplastic anaemia. Br J Haematol. 2006;133: $622-7$.

10. Maciejewski JP, Risitano A, Sloand EM, Nunez O, Young NS. Distinct clinical outcomes for cytogenetic abnormalities evolving from aplastic anemia. Blood. 2002;99:3129-35.

11. Verma A, Deb DK, Sassano A, et al. Cutting edge: activation of the p38 mitogen-activated protein kinase signaling pathway mediates cytokine-induced hemopoietic suppression in aplastic anemia. J Immunol. 2002;168:5984-8.

12. Sloand E, Kim S, Maciejewski JP, Tisdale J, Follmann D, Young NS. Intracellular interferon-gamma in circulating and marrow $\mathrm{T}$ cells detected by flow cytometry and the response to immunosuppressive therapy in patients with aplastic anemia. Blood. 2002;100:1185-91.

13. Takami A, Nakao S, Tatsumi Y, et al. High inducibility of heat shock protein 72 (hsp72) in peripheral blood mononuclear cells of aplastic anaemia patients: a reliable marker of immune-mediated aplastic anaemia responsive to cyclosporine therapy. $\mathrm{Br} \mathrm{J}$ Haematol. 1999;106:377-84.

14. Sloand E, Scheinberg P, Fenlon E, et al. Monosomy 7 detected by FISH at disease presentation is a marker for non-response to immunosuppression. ASH Annu Meet Abstr. 2007;110:1697.

15. Nakao S, Sugimori C, Yamazaki H. Clinical significance of a small population of paroxysmal nocturnal hemoglobinuria-type cells in the management of bone marrow failure. Int $\mathrm{J}$ Hematol. 2006;84:118-22.

16. Scheinberg $\mathrm{P}, \mathrm{Wu} \mathrm{CO}$, Nunez O, Young NS. Predicting response to immunosuppressive therapy and survival in severe aplastic anaemia. Br J Haematol. 2009;144:206-16.

17. Yoshida N, Yagasaki H, Takahashi Y, et al. Clinical impact of HLA-DR15, a minor population of paroxysmal nocturnal haemoglobinuria-type cells, and an aplastic anaemia-associated autoantibody in children with acquired aplastic anaemia. Br J Haematol. 2008;142:427-35.

18. Gluckman E, Devergie A, Poros A, Degoulet P. Results of immunosuppression in 170 cases of severe aplastic anaemia. 
Report of the European Group of Bone Marrow Transplant (EGBMT). Br J Haematol. 1982;51:541-50.

19. Chang MH, Kim KH, Kim HS, et al. Predictors of response to immunosuppressive therapy with antithymocyte globulin and cyclosporine and prognostic factors for survival in patients with severe aplastic anemia. Eur J Haematol. 2010;84:154-9.

20. Afable MG 2nd, Shaik M, Sugimoto Y, et al. Efficacy of rabbit anti-thymocyte globulin in severe aplastic anemia. Haematologica. 2011;96:1269-75.

21. Yoshida N, Yagasaki H, Hama A, et al. Predicting response to immunosuppressive therapy in childhood aplastic anemia. Haematologica. 2011;96:771-4.

22. Olnes MJ, Scheinberg P, Calvo KR, et al. Eltrombopag and improved hematopoiesis in refractory aplastic anemia. N Engl $\mathrm{J}$ Med. 2012;367:11-9.

23. Valdez JM, Scheinberg P, Nunez O, Wu CO, Young NS, Walsh TJ. Decreased infection-related mortality and improved survival in severe aplastic anemia in the past two decades. Clin Infect Dis. 2011;52:726-35.

24. Fuhrer M, Rampf U, Baumann I, et al. Immunosuppressive therapy for aplastic anemia in children: a more severe disease predicts better survival. Blood. 2005;106:2102-4.

25. Scheinberg P, Wu CO, Nunez O, Young NS. Long-term outcome of pediatric patients with severe aplastic anemia treated with antithymocyte globulin and cyclosporine. J Pediatr. 2008;153: 814-9.

26. Kojima S, Hibi S, Kosaka Y, et al. Immunosuppressive therapy using antithymocyte globulin, cyclosporine, and danazol with or without human granulocyte colony-stimulating factor in children with acquired aplastic anemia. Blood. 2000;96:2049-54.

27. Fuhrer M, Burdach S, Ebell W, et al. Relapse and clonal disease in children with aplastic anemia (AA) after immunosuppressive therapy (IST): the SAA 94 experience. German/Austrian Pediatric Aplastic Anemia Working Group. Klin Padiatr. 1998;210: 173-9.

28. Huang IA, Jaing TH, Yang CP, et al. Single-Center Experience: immunosuppressive therapy as frontline treatment for 33 children with acquired severe aplastic anemia. Pediatr Hematol Oncol. 2009;26:487-95.

29. Calado RT, Young NS. Telomere maintenance and human bone marrow failure. Blood. 2008;111:4446-55.

30. Calado RT, Young NS. Telomere diseases. N Engl J Med. 2009;361:2353-65.

31. Ball SE, Gibson FM, Rizzo S, Tooze JA, Marsh JC, GordonSmith EC. Progressive telomere shortening in aplastic anemia. Blood. 1998;91:3582-92.

32. Brummendorf TH, Maciejewski JP, Mak J, Young NS, Lansdorp PM. Telomere length in leukocyte subpopulations of patients with aplastic anemia. Blood. 2001;97:895-900.

33. Fogarty PF, Yamaguchi H, Wiestner A, et al. Late presentation of dyskeratosis congenita as apparently acquired aplastic anaemia due to mutations in telomerase RNA. Lancet. 2003;362:1628-30.
34. Yamaguchi H, Calado RT, Ly H, et al. Mutations in TERT, the gene for telomerase reverse transcriptase, in aplastic anemia. N Engl J Med. 2005;352:1413-24.

35. Scheinberg P, Cooper JN, Sloand EM, Wu CO, Calado RT, Young NS. Association of telomere length of peripheral blood leukocytes with hematopoietic relapse, malignant transformation, and survival in severe aplastic anemia. JAMA. 2010;304: 1358-64.

36. Ma H, Zhou Z, Wei S, et al. Shortened telomere length is associated with increased risk of cancer: a meta-analysis. PLoS ONE. 2011;6:e20466.

37. Wentzensen IM, Mirabello L, Pfeiffer RM, Savage SA. The association of telomere length and cancer: a meta-analysis. Cancer Epidemiol Biomarkers Prev. 2011;20:1238-50.

38. Willeit P, Willeit J, Mayr A, et al. Telomere length and risk of incident cancer and cancer mortality. JAMA. 2010;304:69-75.

39. O'Sullivan JN, Bronner MP, Brentnall TA, et al. Chromosomal instability in ulcerative colitis is related to telomere shortening. Nat Genet. 2002;32:280-4.

40. Risques RA, Vaughan TL, Li X, et al. Leukocyte telomere length predicts cancer risk in Barrett's esophagus. Cancer Epidemiol Biomarkers Prev. 2007;16:2649-55.

41. Calado RT, Cooper JN, Padilla-Nash HM, et al. Short telomeres result in chromosomal instability in hematopoietic cells and precede malignant evolution in human aplastic anemia. Leukemia. 2012;26:700-7.

42. Hemann MT, Strong MA, Hao LY, Greider CW. The shortest telomere, not average telomere length, is critical for cell viability and chromosome stability. Cell. 2001;107:67-77.

43. Baird DM, Rowson J, Wynford-Thomas D, Kipling D. Extensive allelic variation and ultrashort telomeres in senescent human cells. Nat Genet. 2003;33:203-7.

44. Dumitriu B, Ueda Y, Kajigaya S, Townsley DM, Young NS. Very short telomeres of peripheral blood leukocytes precede clinical progression to myelodysplasia with monosomy 7 in aplastic anemia patients. ASH Annu Meet Abstr. 2012;120:1265.

45. Gardner FH, Juneja HS. Androstane therapy to treat aplastic anaemia in adults: an uncontrolled pilot study. $\mathrm{Br} \mathrm{J}$ Haematol. 1987;65:295-300.

46. Calado RT, Yewdell WT, Wilkerson KL, et al. Sex hormones, acting on the TERT gene, increase telomerase activity in human primary hematopoietic cells. Blood. 2009;114:2236-43.

47. Chen J, Desierto MJ, Dent JJ, et al. Androgen treatment mitigates hematopoietic cell telomere attrition in vivo. ASH Annu Meet Abstr. 2012;120:516.

48. Ziegler P, Schrezenmeier H, Akkad J, et al. Telomere elongation and clinical response to androgen treatment in a patient with aplastic anemia and a heterozygous hTERT gene mutation. Ann Hematol. 2012;91:1115-20.

49. Townsley DM, Dumitriu B, Kajigaya S, Calado RT, Scheinberg $\mathrm{P}$, Young NS. Clinical and genetic heterogeneity of telomere diseases. ASH Annu Meet Abstr. 2012;120:2373. 